\title{
Algorithms for Global Total Least Squares Modelling of Finite Multivariable Time Series*
}

\author{
BEREND ROORDA $\dagger$ \\ We consider the optimal approximation of an observed multivariable time \\ series by one that satisfies a set of linear, time-invariant difference \\ equations, under a constraint on the number of independent equations and \\ their total lag.
}

Key Words-Identification; least squares approximations; linear systems; state space methods Kalman filters.

\begin{abstract}
In this paper we present several algorithms related to the global total least squares (GTLS) modelling of multivariable time series observed over a finite time interval. A GTLS model is a linear, time-invariant finite-dimensional system with a behaviour that has minimal Frobenius distance to a given observation. The first algorithm determines this distance. We also give a recursive version of this, which is comparable to Kalman filtering. Necessary conditions for optimality are described in terms of state space representations. Further we present a Gauss-Newton algorithm for the construction of GTLS models. An example illustrates the results.
\end{abstract}

\section{INTRODUCTION}

The central problem in time series modelling is to find a reasonably simple model that captures the main characteristics of the data. The dominant approach is to model observations as a relatively simple function of their past with a stochastic deviation added to this, representing the prediction error. In this paper we follow the approximate modelling approach, as introduced in Willems (1986a, b, 1987). The basic idea is to aim for a relatively simple model that explains the largest part of the data, leaving the deviations unexplained. So, instead of making predictive statements of a stochastic nature, we aim for descriptive statements that hold approximately.

In global total least squares (GTLS) modelling a given observation is approximated by a time series that satisfies linear, time-invariant dynamic

* Received 26 January 1994; rcceived in final form 29 July 1994. This paper was not presented at any IFAC meeting. This paper was recommended for publication in revised form by Associate Editor Dr P. Van den Hof under the direction of Editor T. Söderström. Corresponding author Dr B. Roorda. Tel. (31) 10 4088900; Fax (31) 10 4527347; E-mail roorda@tir.few.eur.nl.

† Tinbergern Institute, Erasmus University Rotterdam, Oostmaaslaan 950-952, NL-3063 DM Rotterdam, The Netherlands. relationships. The relationships correspond to a linear, time-invariant finite-dimensional system, which is considered as an approximate model for the observation. The aim is to minimize a total least squares criterion for the approximation error, under restrictions on the number of inputs and states of the model. For an extensive introduction and motivation we refer to Roorda and Heij (1995), where we discussed this problem for square summable time series over the infinite time axis $Z$. In that paper we described an iterative method for determining locally optimal models, derived optimality conditions and gave some simulation results.

In this paper we shall focus on the more practical aspects of this modelling approach. Therefore we discuss the GTLS modelling of finite time series, and pay more attention to algorithmic aspects. In order to make this paper sufficiently self-contained, we first give a brief introduction to GTLS modelling in Sections 2 and 3. In Section 4 we introduce isometric state representations, which play an important role in all algorithms.

The problem of constructing GTLS models is divided in two parts. The first concerns the evaluation of the distance between models and an observation. This is solved in Section 5 by an algorithm that determines the optimal approximation of an observation within the behaviour of a given system. Section 6 contains a recursive version of this algorithm, which can be considered as a Kalman filter for a deterministic system with some degrees of freedom in it, reflecting the effect of unknown inputs. For a description of the Kalman filter we refer to Anderson and Moore (1979).

The second part of the GTLS problem consists in the construction of models with minimal 
distance to a given observation. First, in Section 7 , we derive necessary optimality conditions in terms of state representations, which are derived from an iterative algorithm in Roorda and Heij (1995) for constructing locally optimal models. In Section 8 we present a Gauss-Newton algorithm for determining locally optimal models. This algorithm is easily implemented, and is in general much faster than the previous algorithm.

We conclude by a simple example illustrating the algorithms in this paper.

The proofs of propositions and of the correctness of the algorithms are given in an Appendix.

\section{GTLS FOR FINITE TIME}

Let there be given an observation

$$
w: T \rightarrow \mathrm{R}^{q}, \quad \text { with } T=\{1,2, \ldots, N\} .
$$

Our aim is to model this time series by means of linear, time-invariant, dynamic relationships. Generically, time series do not satisfy any such relationships exactly. One way to model that gap between data and models is to allow for stochastic deviations from the relationships, resulting in a stochastic model. Alternatively, in approximate modelling the observation is approximated by a time series that does satisfy such relations, without an attempt to model this gap stochastically. According to this, the basic scheme for our modelling theory is the decomposition

$$
w=\hat{w}+\tilde{w}
$$

where $\hat{w}$ is required to be regular and such that the corresponding deviation $\tilde{w}$ is as small as possible. A time series is called regular if it satisfies linear time-invariant difference equations of finite lag, i.e.

$$
\begin{aligned}
& r_{d} \hat{w}(t)+\cdots+r_{0} \hat{w}(t-d)=0 \text { for } d+1 \leq t \leq N, \\
& \text { with } r_{i} \in \mathrm{R}^{1 \times q} \text {. }
\end{aligned}
$$

We impose a priori a lower bound $p$ on the number of independent equations of the type (3), and an upper bound on their total lag, i.e. the sum of the lags of the individual equations. Equations are called independent if they cannot be replaced by a smaller equivalent set of equations, i.e. by a set of equations with the same solution set on $\mathrm{Z}$.

The error in approximating the observation $w$ by a regular part $\hat{w}$ is measured by the Frobenius norm of the corresponding deviation $\tilde{w}=w-\hat{w}$, denoted by

$$
\|\tilde{w}\|:=\left[\sum_{t=1}^{N}|\tilde{w}(t)|^{2}\right]^{1 / 2},
$$

where $|\cdot|$ denotes the Euclidean norm on $\mathbf{R}^{q}$.

Let $\mathscr{B}_{T}^{q, m, n}$ denote the set of all time series on the time interval $T \subset \mathrm{Z}$ with $q$ components that satisfy at least $p:=q-m$ independent equations of the type (3) with total lag at most $n$. Then the GTLS problem for finite time series can be formulated as

$$
\min \left\{\|w-\hat{w}\| ; \hat{w} \in \mathscr{B}_{T}^{q, m, n}\right\} .
$$

The name 'global total least squares' is motivated by the fact that we use the total least squares criterion (4), in which deviations in all components of the data are allowed, and weighted similarly by the sum of squares. Further, we approximate observations by global solutions of difference equations, which is in contrast with, for example, prediction error methods that only take into account the one-step-ahead predictions of difference equations at each time separately. For a comparison with other identification methods, such as regression, the output error method and the local total least squares method, we refer to Roorda and Heij (1995).

\section{GTLS MODELS}

In our modelling approach we are not only interested in the optimal approximation of an observation, but also in the corresponding set of difference equations, since they represent its structural properties. These equations can be interpreted as an optimal approximate model for the observation. It is obvious that sets of equations with the same solution set are equivalent, since they yield the same approximation error for every observation. Hence it is not the equations themselves but rather their solution set that is the essential object in our modelling procedure. These solution sets correspond to the behaviour of linear, time-invariant systems with a finite-dimensional state space, abbreviated as 'LTF systems'.

We consider behaviours on the infinite time axis $\mathrm{Z}$, irrespective of the actual observation interval, in order to streamline the theory concerning their representations. Moreover, the behaviour of models outside the observation interval is relevant for predictions and recursive procedures. Therefore, in the sequel, we shall identify systems with their behaviour on $Z$.

Let $\mathrm{B}^{q}$ denote the class of LTF systems with $q$ variables, i.e. with the total number of inputs 
and outputs equal to $q$. The restrictions we impose on the number of equations and their total lag can be translated to restrictions on the dimension of the behaviour of systems on finite time intervals, as follows. Let $\mathscr{B}_{T}$ denote the restriction of a system $\mathscr{B}$ to a time interval $T \subset \mathrm{Z}$, and let $[1, N]$ denote the interval $\{t \in Z ; 1 \leq t \leq N\}$. We define the following concept of complexity of systems, which is based on the fact that the dimension of $\mathscr{B}_{[1, N]}$ grows linearly with $N$ if $N$ is sufficiently large (cf. Willems, 1986a, b, 1987).

Definition 3.1. (Complexity.) The complexity of an LTF system $\mathscr{B}$ is defined as the unique pair of finite numbers $(m, n)$, such that $\operatorname{dim} \mathscr{B}_{[1, N]}=$ $m N+n$ for $N \geq n$. Complexities are partially ordered by $\left(m^{\prime}, n^{\prime}\right) \leq(m, n)$ if $m^{\prime} \leq m$ and $n^{\prime} \leq n$.

The number $m$ represents the degree of freedom at each time instant, while $n$ corresponds to the degree of freedom due to initial conditions. Equivalently, the number $q-m$ equals the number of independent difference equations needed to describe the system, and $n$ is their minimal total lag. In terms of classical state representations with inputs and outputs, $m$ equals the number of inputs and $n$ the minimal state dimension. Note, however, that we restrict the number of inputs $m$, but do not impose a priori a specific decomposition of the $q$ system variables into inputs and outputs.

We define the misfit of a system as follows.

Definition 3.2. (Misfit.) The misfit of a lincar, time-invariant finite-dimensional system $\mathscr{B}$ with respect to a time series $w: T \rightarrow \mathrm{R}^{q}$ is defined as $d(w, \mathscr{B}):=\min _{\hat{w} \in \mathscr{B}_{T}}\|w-\hat{w}\|$.

The GTLS problem (5) can now be formulated in terms of systems as follows. Let $\mathrm{B}^{q, m, n}$ denote the systems in $\mathrm{B}^{q}$ with complexity at most $(m, n)$, i.e. systems that can be described by at least $q-m$ independent difference equations with total lag at most $n$.

Definition 3.3. (GTLS for finite time.) For a given observation $W: T \rightarrow \mathrm{R}^{q}$, and given tolerated complexity $(m, n)$, determine an LTF system $\mathscr{R}^{*} \in \mathrm{B}^{q, m, n}$, such that $d\left(w, \mathscr{B}^{*}\right)=$ $\min _{\mathscr{B} \in \mathrm{B}^{q, m, n}} d(w, \mathscr{B})$.

This involves a double minimization. The inner minimization, evaluating the misfit $d(w, \mathscr{B})$, amounts to optimization over a linear space, and is addressed in Sections 5 and 6. Secondly, we have to determine a system for which this misfit is minimal. This is a non-linear optimization problem over a non-convex set, for which we present iterative algorithms in Sections 7 and 8.

\section{ISOMETRIC STATE REPRESENTATIONS}

One of the basic questions in GTLS modelling is how to calculate the misfit of a system with respect to a given observation (cf. Definition 3.2). Obviously this requires a numerical representation of the system. In this section we develop a type of representation that is extremely useful for this purpose, namely the isometric state representations (ISRs). Moreover, they also play an important role in the Gauss-Newton algorithm for determining locally optimal models.

Definition 4.1. (Isometric state representation.) A state representation $(A, B, C, D)$ of a system $\mathscr{B} \in \mathrm{B}^{q}$ is a description of the form

$$
\begin{aligned}
& \mathscr{B}=\left\{\hat{w}: Z \rightarrow \mathrm{R}^{q} ; \exists \hat{x}: \mathrm{Z} \rightarrow \mathrm{R}^{n}, \hat{v}: \mathrm{Z} \rightarrow \mathrm{R}^{m},\right. \\
& \text { such that } \hat{x}(t+1)=A \hat{x}(t)+B \hat{v}(t) \\
&\hat{w}(t)=C \hat{x}(t)+D \hat{v}(t) \forall t \in Z\},
\end{aligned}
$$

with $A \in \mathbf{R}^{n \times n}, B \in \mathbf{R}^{n \times m}, C \in \mathbf{R}^{q \times n}, D \in \mathbf{R}^{q \times m}$. It is called isometric if, moreover,

$$
\left[\begin{array}{ll}
A & B \\
C & D
\end{array}\right]^{\mathrm{T}}\left[\begin{array}{cc}
A & B \\
C & D
\end{array}\right]=\left[\begin{array}{cc}
I_{n} & 0 \\
0 & I_{m}
\end{array}\right]
$$

Here $\hat{v}$ is an auxiliary input, $\hat{x}$ is a state trajectory and $\hat{w}$ is a system trajectory, $m$ denotes the number of auxiliary inputs and $n$ the number of state variables. The pair $(m, n)$ is called the dimension of the representation. The system defined by this representation is denoted by $\mathscr{B}(A, B, C, D)$. Representations are equivalent if they describe the same behaviour. They are called minimal if they have minimal dimension, i.e. if the number of state variables and the number of auxiliary inputs are both minimal. Systems in $\mathrm{B}^{q, m, n}$ are precisely those systems that admit an SR with dimension $(m, n)$. The complexity of a system equals the dimension of a minimal SR (see Willems, 1986a, b, 1987).

The isometry property (6) can be imposed almost without loss of generality, which is made precise in the following proposition. We call a system stabilizable if all trajectories on finite time intervals can be made to converge to zero in the future. This correponds to the classical definition in terms of inputs and states, imposing that the state in a minimal input/state/output 
representation can be made to converge to zero by choosing appropriate inputs.

Proposition 4.2. (Existence of ISR.) A stabilizable LTF system admits an isometric minimal state representation.

For this reason, we shall restrict our attention to stabilizable systems. This is only a minor restriction, since stabilizability is a generic property of LTF systems.

We list some properties of ISRs that are used in the sequel.

Proposition 4.3. (Properties of ISR.) Let $(A, B, C, D)$ denote an isometric state representation of a stabilizable system $\mathscr{B}$.

(1) The matrix $A$ is stable, i.e. $\|A x\| \leq\|x\|$. If the representation is minimal then $A$ is asymptotically stable.

(2) Two minimal ISRs $(A, B, C, D)$ and $\left(A^{\prime}, B^{\prime}, C^{\prime}, D^{\prime}\right)$ are equivalent if and only if there exist unitary matrices $U$ and $V$ such that

$\left(A^{\prime}, B^{\prime}, C^{\prime}, D^{\prime}\right)=\left(U A U^{\mathrm{T}}, U B V, C U^{\mathrm{T}}, D V\right)$.

(3) Let $\hat{x}$ and $\hat{v}$ denote, respectively, the state and auxiliary input corresponding to $\hat{w} \in \mathscr{B}_{T}$ with $T=\{1, \ldots, N\}$, i.e. $\hat{x}(t+1)=A \hat{x}(t)+$ $B \hat{v}(t), \hat{w}(t)=C \hat{x}(t)+D \hat{v}(t)$ for $t \in T$. Then

$$
\|\hat{w}\|^{2}=\|\hat{v}\|^{2}+|\hat{x}(1)|^{2}-|\hat{x}(N+1)|^{2} .
$$

\section{OPTIMAL APPROXIMATION WITHIN A SYSTEM}

In this section we consider the first part of the GTLS problem (Definition 3.3), namely the elevation of the misfit (Definition 3.2). Moreover, we shall determine the optimal approximation within the behaviour of a given system, for which this misfit is achieved.

The algorithm is based on the following considerations. As we consider linear behaviours $\mathscr{B}$, the original approximation of an observation $w$ on a time interval $T$ is given by its orthogonal projection onto $\mathscr{B}_{T}$. This implies that the optimal approximation corresponds to a decomposition of an observation into a part contained in $\mathscr{B}_{T}$ and a part in its orthogonal complement $\left(\mathscr{B}_{T}\right)^{\perp}$. Clearly, such a decomposition is unique. The key result behind the algorithm is contained in the following proposition. Let $\left(\mathscr{R}_{T}\right)^{\perp}$ denote the orthogonal complement of $\mathscr{B}_{T}$, i.e. $\left(\mathscr{B}_{T}\right)^{\perp}:=$ $\left\{\tilde{w}: T \rightarrow \mathrm{R}^{q} ;\langle w, \tilde{w}\rangle=0\right.$ for all $\left.w \in \mathscr{B}_{T}\right\}$, where the inner product is defined by $\langle w, \tilde{w}\rangle:=$ $\sum_{t \in T} w(t)^{\mathrm{T}} \tilde{w}(t)$

Proposition 5.1. (Orthogonal complement.) Let $(A, B, C, D)$ denote an ISR of an LTF system $\mathscr{B}$, and let $\tilde{B}$ and $\tilde{D}$ be such that $\left[\begin{array}{ccc}A & B & \tilde{B} \\ C & D & \tilde{D}\end{array}\right]$ is a unitary matrix. Then

$$
\begin{array}{r}
\left(\mathscr{B}_{T}\right)^{\perp}=\left\{\tilde{w}: T \rightarrow R^{q} ;\{\ldots, 0,0, \tilde{w}, 0,0, \ldots\}\right. \\
\in \mathscr{B}(A, \tilde{B}, C, \tilde{D})\} .
\end{array}
$$

With some slight abuse of terminology, we shall call $(A, \tilde{B}, C, \tilde{D})$ an ISR of the orthogonal complement of $\mathscr{B}$. It turns out that the optimal decomposition of an observation $w$ over $\mathscr{B}_{T}$ and $\left(\mathscr{B}_{T}\right)^{\perp}$ can be constructed by subsequently one backward and one forward iteration in terms of the ISR of $\mathscr{B}$ and its orthogonal complement, as follows.

Algorithm 1. (Optimal approximation in a given system.)

Data:

- an observation $w: T \rightarrow \mathrm{R}^{q}, T=\{1, \ldots, N\}$;

- a stabilizable system $\mathscr{B}$ with isometric minimal state representation $(A, B, C, D)$.

Step 1. Determine $\tilde{B}$ and $\tilde{D}$ such that $\left[\begin{array}{lll}A & B & \tilde{B} \\ C & D & \tilde{D}\end{array}\right]$ is a unitary matrix.

Step 2. Define $\hat{v}, \tilde{v}$ and $x$ by the backward recursive equations

$$
\begin{aligned}
x(t) & =A^{\mathrm{T}} x(t+1)+C^{\mathrm{T}} w(t), \\
\hat{v} & =B^{\mathrm{T}} x(t+1)+D^{\mathrm{T}} w(t), \\
\tilde{v} & =\tilde{B}^{\mathrm{T}} x(t+1)+\tilde{D}^{\mathrm{T}} w(t),
\end{aligned}
$$

with the end state $x(N+1)$ chosen such that $\|\tilde{\boldsymbol{v}}\|$ is minimal.

Step 3. Define $\hat{w}$ by

$$
\begin{aligned}
\hat{x}(t+1) & =A \hat{x}(t)+B \hat{v}(t), \quad \text { with } \hat{x}(1)=x(1), \\
\hat{w}(t) & =C \hat{x}(t)+D \hat{v}(t),
\end{aligned}
$$

and $\tilde{w}$ by

$$
\begin{aligned}
\tilde{x}(t+1) & =A \tilde{x}(t)+\tilde{B} \tilde{v}(t), \quad \text { with } \tilde{x}(1)=0, \\
\tilde{w}(t) & =C \tilde{x}(t)+\tilde{D} \tilde{v}(t) .
\end{aligned}
$$

Result:

- $\hat{w}$ is the optimal approximation of $w$ in $\mathscr{B}$;

- $\tilde{w}=w-\hat{w}$ is the corresponding approximation error;

- $d(w, \mathscr{B})-\|\tilde{w}\|-\mid \tilde{v} \|$

As mentioned in Section 1, the proof of correctness is given in the Appendix.

Remark. Note that the Definition 3.2 of the misfit criterion is symmetric in time, since $d(w, \mathscr{B})=d\left(w_{\mathrm{r}}, \mathscr{B}_{\mathrm{r}}\right), \quad$ with $\quad w_{\mathrm{r}}(t):=w(-t) \quad$ and 
$\mathscr{B}_{\mathrm{r}}:=\left\{w ; w_{r} \in \mathscr{R}\right\}$. However, Proposition 5.1 and Algorithm 1 are not time symmetric, since they are in terms of ISRs that describe the forward evolution of the state. Dual (time-reversed) counterparts of the results can be obtained by using backward ISRs $\left(A_{\mathrm{r}}, B_{\mathrm{r}}, C_{\mathrm{r}}, D_{\mathrm{r}}\right)$, which satisfy (6) and represent the system $\{w: Z \rightarrow$ $\mathrm{R}^{q} ; \exists x, v$ such that $x(t)=A_{r} x(t+1)+B_{r} v(t)$, $w(t)=C_{r} x(t+1)+1+D_{r} v(t) \forall t \in Z$ \} . Such representations exist for antistabilizable systems, i.e. systems that are stabilizable for the timereversed direction. They can be obtained by first transforming an SR $(A, B, C, D)$ with $A$ invertible to a backward SR $\left(A^{-1}\right.$, $\left.-A^{-1} B, C A^{-1}, D-C A^{-1} B\right)$, and then applying the same construction as for forward SRs to obtain an equivalent isometric one.

\section{RECURSIVE PROJECTION}

In this section we describe a recursive version of the projection algorithm described in Section 5. This makes explicit the effect of a new observation on the optimal approximation of its past in a given system.

We use the following notation. Let $\mathscr{B}$ denote a given LTF system with ISR $(A, B, C, D)$, and let $\widetilde{B}$ and $\tilde{D}$ be defined as in Algorithm 1. The observation interval is given by $\{1, \ldots, N\}$. Further we define:

- $\hat{w}_{t}$ as the optimal approximation within $\mathscr{B}$ of the observation up to an including time $t$

- $\tilde{w}_{t}$ as the approximation error corresponding to $\hat{w}_{t}$

- $\hat{x}_{t}, \hat{v}_{t}$ as the state and auxiliary input corresponding to $\hat{w}_{t}$ in $(A, B, C, D)$;

- $\tilde{x}_{t}, \tilde{v}_{t}$ as the state and auxiliary input corresponding to $\tilde{w}_{l}$ in $(A, \tilde{B}, C, \tilde{D})$;

- $x_{t}=\hat{x}_{t}+\tilde{x}_{t}$.

Note that $\hat{w}_{t}, \tilde{w}_{t}, \hat{v}_{t}$ and $\tilde{v}_{t}$ are time series defined on $\{1, \ldots, t\}$, while $\hat{x}_{t}, \tilde{x}_{t}$ and $x_{t}$ are also defined for time $t+1$.

In this notation, the optimal approximation determined in Algorithm 1 is denoted by $\hat{w}_{N}$ and its corresponding state by $\hat{x}_{N}$. In the following algorithm we determine the values for $\hat{x}_{t}(t+1)$ and $\hat{w}_{t}(t)$ recursively for $t=1, \ldots, N$. Comparing $\hat{w}_{t}\left(t^{\prime}\right)$ and $\hat{w}_{N}\left(t^{\prime}\right)$, both are approximations of $w\left(t^{\prime}\right)$, where the former is optimal given the observations up to time $t$, while the latter is based on the whole observation. Stated according to the common terminology in the literature, Algorithm 1 determines the smoothed values of the optimal approximation and the corresponding state, while the next algorithm computes their past induced values.
The algorithm is based on the following considerations. Suppose we have observed a time series $\{w(1), \ldots, w(t-1)\}$ and determined its optimal approximation in $\mathscr{B}$

$w\left(t^{\prime}\right)=\hat{w}_{t-1}\left(t^{\prime}\right)+\tilde{w}_{t-1}\left(t^{\prime}\right)$ for $t^{\prime} \in\{1, \ldots, t-1\}$,

where $\hat{w}_{t-1} \in \mathscr{B}_{[1, t-1]}$ with final state $\hat{x}_{t-1}(t)$. First consider the case where the next observation is a propagation of $\hat{w}_{t-1}$ within $\mathscr{B}$, i.e. $w(t)=$ $C \hat{x}_{t-1}(t)+D \hat{v}(t) \quad$ for some $\hat{v}(t) \in \mathbf{R}^{m}$. Obviously, this will not increase the misfit, since there is no reason to change the approximation before $t$, and no approximation error has to be made at $t$.

Next suppose that $w(t)$ is not compatible with $\hat{w}_{t-1}$ in $\mathscr{B}$, i.e. $\epsilon(t):=w(t)-C \hat{x}_{t-1}(t) \notin \operatorname{im} D$. Then we have to approximate the observation by

$$
\hat{w}_{t}(t)=C \hat{x}_{t}(t)+D \hat{v}_{t}(t),
$$

where $\hat{x}_{t}(t)$ and $\hat{v}_{t}(t)$ have to be determined such that the increase of the misfit, denoted by $m(t)$, is as small as possible. This increase consists of two parts:

- the approximation error at time $t$, i.e. $\left|w(t)-\hat{w}_{t}(t)\right|$, denoted by $m_{0}(t)$;

- an increase of misfit over the past, denoted by $m_{-}(t)$, due to changing the state $\hat{x}_{t-1}(t)$, which is optimal for $\{w(1), \ldots, w(t-1)\}$, into $\hat{x}_{t}(t)$.

In general, keeping the past approximation $\hat{w}_{t-1}$ fixed, and hence keeping $\hat{x}_{t-1}(t)$ fixed, would lead to a large approximation error at time $t$, while minimizing the error at $t$ alone would lead to a large increase in the misfit over the past due to the large change in the state at time $t$. The optimal strategy is a trade-off between the two approaches. The main result underlying the algorithm is that the only feature of the past observations that determines this trade-off is the final state $\hat{x}_{t-1}(t)$ of the optimal approximation. Equivalently, given $\hat{x}_{t-1}(t)$, the optimal values for $\hat{x}_{t}(t)$ and $\hat{v}_{t}(t)$ in (10) are independent of the past observations. This opens the way to compute the optimal values for $\hat{x}_{t}(t+1)$ and $\hat{v}_{t}(t)$ in (10) recursively, although it takes some effort to obtain the correct formulas. The algorithm below is followed by some remarks that might be helpful for understanding the algorithm.

Algorithm 2. (Recursive approximation in a given system.)

Data:

- an observation $w$ on the time interval $T=\{1, \ldots, N\}$

- a stabilizable LTF system $\mathscr{B}$ with minimal ISR $(A, B, C, D)$. 
Step 1. Define $\tilde{W}_{t}, \tilde{F}_{t}, \tilde{G}_{t}$ and $\tilde{H}_{t}$ for $t \in T$ by the equations

$$
\begin{aligned}
\tilde{W}_{t+1} & =A \tilde{W}_{t} A^{\mathrm{T}}+\tilde{B} \tilde{B}^{\mathrm{T}}, \text { with } \tilde{W}_{1}=0, \\
\tilde{F}_{t} & =A \tilde{W}_{t} C^{\mathrm{T}}+\tilde{B} \tilde{D}^{\mathrm{T}}, \\
\tilde{G}_{t} & =C \tilde{W}_{t} C^{\mathrm{T}}+\tilde{D} \tilde{D}^{\mathrm{T}}, \\
\tilde{H}_{t} & =\tilde{G}_{t}-\tilde{F}_{t}^{\mathrm{T}} \tilde{W}_{t+1}^{-1} \tilde{F}_{t} .
\end{aligned}
$$

If the inverse does not exist, replace by a pseudo-inverse.

Step 2. Define for $t \subset T$

$$
\begin{gathered}
\epsilon(t)=w(t)-C \hat{x}_{t-1}(t) \\
\hat{x}_{t}(t+1)=A \hat{x}_{t-1}(t)-\tilde{W}_{t+1}^{-1} \tilde{F}_{t} \epsilon(t), \\
\quad \text { with } \hat{x}_{0}(1)=0, \\
\hat{w}_{t}(t)=C \hat{x}_{t}(t)+\left(I-\tilde{H}_{t}\right) \epsilon(t)
\end{gathered}
$$

and further

$$
\begin{gathered}
m(t)^{2}=\epsilon(t)^{\mathrm{T}} \tilde{H}_{t} \epsilon(t), \\
m_{0}(t)^{2}=\epsilon(t)^{\mathrm{T}} \tilde{H}_{t}^{2} \epsilon(t), \\
m_{-}(t)^{2}=\epsilon(t)^{\mathrm{T}}\left(\tilde{H}_{t}-\tilde{H}_{t}^{2}\right) \epsilon(t) .
\end{gathered}
$$

Result:

- $\hat{x}_{t+1}(t)$ is the end state of the optimal approximation of $\{w(1), \ldots, w(t)\}$ in $\mathscr{B}$;

- $\hat{w}_{t}(t)$ is the corresponding optimal approximation at time $t$ :

- $m(t)^{2}$ is the increase in squared misfit due to observation $w(t)$;

- $m(t)^{2}=m_{-}(t)^{2}+m_{0}(t)^{2}$, where $m_{-}(t)^{2}$ denotes the increase in squared misfit due to observation $w(t)$ on the past $\{1, \ldots, t-1\}$, and $m_{0}(t)$ the misfit at $t$.

Concerning the interpretation of this algorithm, we make the following remarks:

- The matrix $\tilde{W}_{t}$ can be considered as the finite-time controllability Gramian corresponding to $(A, \tilde{B})$. It measures the costs of changing the optimal end state $\hat{x}_{t-1}(t)$, which is made precise in the proof.

- In order to relate the results to Kalman filtering, which was developed originally in a stochastic context, onc might comparc $\varepsilon(t)$ with an innovation process, representing the unexpected part of a new observation $w(t)$ given the final state $\hat{x}_{t-1}(t)$ of the approximation $\hat{w}_{t-1}$. However, the model $\mathscr{B}$ leaves some degrees of freedom for $\epsilon$, which will be explained below.

- The matrix $\tilde{W}_{t+1}^{-1} \tilde{F}_{t}$ in the second step plays the role of what is called the Kalman gain, indicating the weights by which the deviations $\epsilon(t)$ influence the state evolution.

- The matrix $\tilde{H}_{r}$ can be compared with the inverse covariance matrix of the innovation process (cf. (14)).

Note, however, that our results are only based on the GTLS criterion, without making any stochastic assumptions. It turns out that the inverse covariance matrix $\tilde{H}_{t}$ is singular, and hence its inverse does not exist. This reflects the fact that $\mathscr{B}$ contains some input components, i.e. components whose value can be chosen arbitrarily in $\mathscr{B}$, which would lead to an infinite covariance matrix in a stochastic context. In fact, it is easily verified that $\widetilde{H}_{t} D=0$, corresponding to the fact that $\epsilon(t) \in \operatorname{im} D$ is in perfect correspondence with the system laws. This means that the model 'predicts' that the next observation $w(t)$ will be in an affine space $C \hat{x}_{t}(t)+\operatorname{im} D$, and the level curves of the GTLS criterion consist of elliptic 'cones' around this space. We refer to Section 9 for an illustration of this fact.

Further note that the involved system matrices in Step 2 are time dependent, but independent of the observation. As $N \rightarrow \infty, \tilde{W}_{t}$ converges to the controllability Gramian $\tilde{W}$ of $(A, \tilde{B})$, which is well defined since $A$ is asymptotically stable (cf. Proposition 4.3(1)) Consequently, if $\tilde{W}_{t}$ has become equal to $\tilde{W}$ within a sufficiently high precision level, it can be replaced by $\tilde{W}$, resulting in a time-invariant filter for the optimal approximation in Step 2.

\section{OPTIMALITY CONDITIONS}

In the previous sections we described how to evaluate the misfit of a system with respect to an observation. In this and the following section we discuss the second part of the GTLS problem (Definition 3.3), namely determining a system with minimal misfit with respect to a given observation. Formulated in terms of state representations, this amounts to the following.

Definition 7.1. (GTLS in terms of $S R$.) For given observation $\{w(1), \ldots, w(N)\}$, and given tolerated complexity $(m, n)$, determine a state representation $(A, B, C, D)$ with $m$ auxiliary inputs and $n$ states, an auxiliary input $\{\hat{v}(1), \ldots, \hat{v}(N)\}$ and initial state $\hat{x}(1) \in \mathbf{R}^{n}$, such that for $\hat{w}(t):=C A^{t-1} \hat{x}(1)+D \hat{v}(t)+$ $\sum_{k=1}^{t-1} C A^{k-1} B \hat{v}(k)$, the error $\|w-\hat{w}\| \quad$ is minimal.

First we briefly describe a slightly modified version of the algorithm in Roorda and Heij (1995), mainly because we can derive from it some necessary conditions for optimality. In fact, 
these conditions form a characterization of stationary points with respect to the GTLS criterion. We call a system $\mathscr{B}$ a stationary point for an observation $w$ if all the derivatives of the GTLS misfit $d(w, \mathscr{B}(A, B, C, D))$ with respect to the system parameters are zero for a minimal SR of $\mathscr{B}$. In the next section we present a Gauss-Newton algorithm, which is in general much faster.

\section{Algorithm 3. (Model improvement algorithm.)}

Data:

- an observation $w: T \in \mathrm{R}^{q}, T=\{1, \ldots, N\}$;

- an isometric state representation $(A, B, C, D)$ with $m$ auxiliary inputs and $n$ states, corresponding to an initial model $\mathscr{B} \in \mathrm{B}^{q, m, n}$.

Step 1. Determine optimal $\hat{v}$ and $\hat{x}(1)$ by Algorithm 1.

Step 2. Determine optimal $C$ and $D$, and transform the result to an equivalent ISR.

Step 3. Determine optimal $B, D$ and $\hat{x}(1)$, and transform the result to an equivalent ISR.

Step 4. Determine optimal $B, D$ and $\hat{v}$ in an ISR, i.e. such that (6) remains valid, and redefine $\mathscr{B}$ as the system corresponding to the resulting ISR.

Step 5. If the misfit has decreased, go to Step 1, else stop.

Result. $\mathscr{B}$ is a stationary point with respect to the GTLS critcrion.

Of course, in practice the algorithm is stopped when the decrease of misfit has come below a certain threshold, depending on the required accuracy. The name of the algorithm is based on the fact that Steps 2-4 all improve the initial model, unless it is a stationary point. Further, we remark that there is no theoretical or practical reason for the order of these steps.

The following optimality conditions are derived from the consideration that for optimal models the algorithm can give no improvement. In fact, they give a characterization of the stationary points of the GTLS criterion. These optimality conditions will be derived for controllable systems, i.e. systems for which each trajectory can be steered to zero within finite time. This is equivalent to the condition that there exists an SR with $(A, B)$ controllable. We remark that we conjecture that the following results are valid for the non-generic class of non-controllable systems as well, but we have to leave this issue as a point for further research.

We express the optimality conditions in terms of empirical covariances. For two sequences $a:\{1, \ldots, N\} \rightarrow \mathrm{R}^{k}, \quad b:\{1, \ldots, N\} \rightarrow \mathrm{R}^{l}$ this is defined as $\operatorname{cov}(a, b):=\sum_{t=1}^{N} a(t) b(t)^{\mathrm{T}} \in \mathbf{R}^{k \times t}$. Further, $\operatorname{cov}(\sigma a, b)$ is defined as $\sum_{t=1}^{N-1} a(t+$ 1) $b(t)^{\mathrm{T}}$, and $\left[a_{1}, a_{2}\right]$ denotes the combined trajectory $\left[\begin{array}{ll}a_{1}^{\mathrm{T}} & a_{2}^{\mathrm{T}}\end{array}\right]^{\mathrm{T}}$.

Theorem 7.2. (Optimality conditions.) Let $\mathscr{B}$ denote a controllable GTLS model for an observation $w: T \rightarrow \mathrm{R}^{q}$ with $T=\{1, \ldots, N\}$. Let $\hat{w} \in \mathscr{B}$ denote the optimal approximation of $w$, and $\tilde{w} \in\left(\mathscr{B}_{T}\right)$ the corresponding approximation error. Let $\hat{x}$ and $\hat{v}$ denote, respectively, the state and auxiliary input corresponding to $\hat{w}$ in a minimal state representation of $\mathscr{B}$, and let $\tilde{x}$ and $\tilde{v}$ be defined analogously for $\tilde{w}$. Then the following equivalent conditions hold:

(1) $\operatorname{cov}(\hat{v}, \tilde{v})=0$ $\operatorname{cov}(\hat{x}, \tilde{v})=0$

and

(2) $\operatorname{cov}([\hat{v}, \hat{x}],[\tilde{v}, \tilde{x}])=0$

(3) $\operatorname{cov}([\hat{v}, \hat{x}, \hat{w}, \sigma \hat{x}],[\tilde{v}, \tilde{x}, \tilde{w}, \sigma \tilde{x}])=0$.

So, roughly speaking, the optimality of a model with respect to the GTLS criterion results in the complete absence of correlation between the approximation, its state and its auxiliary input on the one hand, and the error, its state and auxiliary input on the other. We refer to Roorda and Heij (1995) for the use of these optimality conditions in measuring the accuracy of the optimality of a model without knowing the optimal model itself.

\section{A GAUSS-NEWTON ALGORITHM FOR GTLS}

In this section we present a Gauss-Newton algorithm for the construction of stationary points of the GTLS criterion. As already mentioned in the previous section, it is in general much faster than Algorithm 3. Moreover, the results on system representations underlying this algorithm might be of interest on their own.

The algorithm is based on a formulation of the GTLS problem in terms of ISRs, derived from Algorithm 1. In this section we use the following notation:

- $w$ is a given observation on the time interval $\{1, \ldots, N\}$ with $q$ components;

- $(m, n)$ denotes the tolerated complexity;

- $(A, B, C, D)$ denotes an ISR with dimension $(m, n)$, i.e. with $m$ auxiliary inputs and $n$ state variables;

- $\mathscr{I}$ is the space of ISRs with dimension $(q-m, n)$;

- elements $(A, \tilde{B}, C, \tilde{D}) \in \mathscr{I}$ will be as- 
sociated with the orthogonal complement of models $\mathscr{B}(A, B, C, D)$ as described in Proposition 5.1;

- $u_{k}$ denotes the space of $k \times k$ unitary matrices. Hence

$$
\left[\begin{array}{ccc}
A & B & \tilde{B} \\
C & D & \tilde{D}
\end{array}\right] \in \mathcal{U}_{n+q} ;
$$

- $T_{M} \mathscr{I}$ is the tangent space of $\mathscr{I}$ in the ISR $M \in \mathscr{F}$. Elements $\Delta_{M} \in T_{M} \mathscr{I}$ are called a variation of $M$ in the direction of other ISRs, or simply a variation of $m$. Similarly, elements of $T_{M} U_{k}$ are called variations of $M$ in the direction of other unitary matrices.

Depending on the context, $(A, \tilde{B}, C, \tilde{D})$ will denote either a specific ISR related to a given initial model, or the argument of a function on I. The GTLS problem (Definitions 3.3 and 7.1) can now be reformulated as follows.

Proposition 8.1. (GTLS as minimization over ISRs.) Define for a given observation $w$ the function $H: \mathscr{I} \times \mathrm{R}^{n} \rightarrow\left(\mathrm{R}^{q-m)}\right)^{N}$ by

$$
H\left((A, \tilde{B}, C, \tilde{D}), x_{N+1}\right)=\{\tilde{v}(1), \ldots, \tilde{v}(N)\},
$$

with $\tilde{v}(t)$ defined according to (7) by

$$
\begin{aligned}
& x(t)=A^{\mathrm{T}} x(t+1)+C^{\mathrm{T}} w(t), \quad x(N+1)=x_{N+1} \\
& \tilde{v}(t)=\tilde{B}^{\mathrm{T}} x(t+1)+\tilde{D}^{\mathrm{T}} w(t) .
\end{aligned}
$$

The construction of a GTLS model for $w$ amounts to determining $(A, \tilde{B}, C, \tilde{D}) \in \mathscr{I}$ and $x_{N+1} \in \mathrm{R}^{n}$ for which $\|\tilde{\boldsymbol{v}}\|$ is minimal.

A Gauss-Newton algorithm for determining stationary points of the function $H$ has the following structure:

(1) Choose initial values in the domain of $H$, i.e. choose an ISR $M:=(A, \tilde{B}, C, \tilde{D}) \in \mathscr{I}$ and a value for the end state $x_{N+1} \in \mathrm{R}^{n}$. Let $H_{0}$ denote the value of $H$ at the initial point.

(2) Determine how $\tilde{v}$ varies if the initial values are varied, i.e. if the initial ISR is varied in the direction of another ISR, and if the end state is varied. This amounts to determining the derivative $H^{\prime}$ of $H$ at the initial point, or, in geometrical terms, determining the tangent space of im $H$ in $H_{0}$.

(3) Determine the optimal variation of the initial ISR and end state on basis of this derivative, i.e. determine $\left(\Delta_{A}, \Delta_{\tilde{B}}, \Delta_{C}, \Delta_{\tilde{D}}\right)$ and $\bar{x}_{N+1}$ that minimizes $\| H_{0}+H^{\prime}\left(\left(A+\Delta_{A}\right.\right.$, $\left.\tilde{B}+\Delta_{\tilde{B}}, \quad\left(+\Delta_{C}, \tilde{D}+\Delta_{\tilde{D}}\right), \quad x_{N+1}+\bar{x}_{N+1}\right) \|$. Stated in geometrical terms, determine the optimal variation on the basis of the tangent space of $\operatorname{im} H$ as an approximation of $\operatorname{im} H$ in $H_{0}$.

(4) If the resulting model is equal to the initial one, it is a stationary point, so stop. If the resulting model improves the previous one, repeat the algorithm for this model, otherwise first halve the size of the variation repeatedly until it corresponds to a better model.

As compared with the standard Gauss-Newton algorithm, there are two aspects that deserve additional attention.

- The domain of $H, \mathscr{I} \times \mathrm{R}^{n}$, is determined by a non-linear restriction (cf. (17)).

- ISRs for a given system are not unique (cf. Proposition 4.3).

Before we give the implementation of the algorithm sketched above, we first indicate how we handle these two issues. Concerning the first point, we have to analyse how to vary an ISR into the direction of other ISRs. For expository reasons, we not only consider variations of $(A, \tilde{B}, C, \tilde{D})$ but also the corresponding variations of $(A, B, C, D)$. So this amounts to the question of how to vary a unitary matrix as given in (17) into the direction of another unitary one, which is answered in the following lemma.

Lemma 8.2. The tangent space of the space of unitary matrices $U$ in a point $M$ is given by $T_{M} \mathcal{U}=\left\{M K ; K+K^{\mathrm{T}}=0\right\}$.

Note that for a variation $M K \in T_{M} \mathcal{U}$, the matrix $M+M K$ is unitary up to a quadratic term in $K$, since $(M+K)^{\mathrm{T}}(M+K)=I+K^{\mathrm{T}} K$. Although the quadratic term is negligible if $K$ is sufficiently small, this implies that the state representation corresponding to $M+K$ is no longer exactly isometric. Therefore, after computing the optimal variation in Step 3 of the Gauss-Newton algorithm, we have to transform the resulting state representation to an equivalent ISR.

The non-uniqueness of ISRs, mentioned in the second point, will be exploited to decrease the number of variations that we have to take into account in the Gauss-Newton algorithm. More precisely, we shall make use of the fact that, without loss of generality, we can restrict our attention to variations of (17) of the form

$$
\begin{aligned}
{\left[\begin{array}{lll}
\Delta_{A} & \Delta_{B} & \Delta_{\bar{B}} \\
\Delta_{C} & \Delta_{D} & \Delta_{\bar{D}}
\end{array}\right] } & \\
& =\left[\begin{array}{lll}
A & B & \tilde{B} \\
C & D & \tilde{D}
\end{array}\right]\left[\begin{array}{ccc}
0 & L & P \\
-L^{\mathrm{T}} & 0 & Q \\
-P^{\mathrm{T}} & -Q^{\mathrm{T}} & 0
\end{array}\right] .
\end{aligned}
$$


This means that for all variations as described in Lemma 8.2, there exists an equivalent variation corresponding to zero-diagonal blocks of $K$. Moreover, if $(A, B, C, D)$ is minimal, different variations of this form are not equivalent, i.e. they all correspond to changes into the direction of different systems, which means that generically we have taken full account of the non-uniqueness of ISRs in this way.

Summarizing, we shall prove that for the implementation of the Gauss-Newton algorithm, it suffices to consider variations of ISRs as given by (20), and, since the corresponding SRs are no longer exactly isometric, we have to transform them back to isometric ones in each iteration. The steps of the following algorithm are numbered according to the structure as indicated above.

\section{Algorithm 4. (Gauss-Newton for GTLS.) Data:}

- an observation $w: T \rightarrow \mathrm{R}^{q}, T=\{1, \ldots, N\}$;

- a bound $(m, n)$ for the model complexity.

Step 1. Choose an initial isometric state representation $(A, \tilde{B}, C, \tilde{D}) \in \mathscr{I}$ and determine $B$ and $D$ such that $\left[\begin{array}{lll}A & B & \tilde{B} \\ C & D & \tilde{D}\end{array}\right]$ is a unitary matrix. Construct $x, \hat{v}$ and $\tilde{v}$ according to Algorithm 1. This gives an initial value for $x_{N+1}$ that is optimal for the chosen initial ISR, so that $\tilde{v}=H\left(M, x_{N+1}\right)=: H_{0}$.

Step 2. The derivative $\bar{v}:=H^{\prime}\left(\Delta_{M}, \bar{x}_{N+1}\right)$ of $H$ in $\left(M, x_{N+1}\right)$ in direction $\Delta_{M}:=\left(\Delta_{A}, \Delta_{\tilde{B}}, \Delta_{C}, \Delta_{\tilde{D}}\right)$ of the form (20) is given by

$$
\begin{aligned}
\bar{x}(t)=A^{\mathrm{T}} \bar{x}(t+1)+L \hat{v}(t)+P \tilde{v}(t), \\
\text { with } \bar{x}(N+1)=\bar{x}_{N+1}, \\
\bar{v}(t)=\tilde{B}^{\mathrm{T}} \bar{x}(t+1)-P^{\mathrm{T}} x(t)-Q^{\mathrm{T}} \hat{v}(t) .
\end{aligned}
$$

Define

$$
\mathscr{T}:=\left\{\bar{v} ; \exists L, P, Q, \bar{x}_{N+1} \text { such that } \bar{v} \text { satisfies }(21)\right\},
$$

which can be considered as the relevant part of the tangent space of im $H$ in $\tilde{v}$.

Step 3. Determine the variation $\left(\Delta_{M}, \bar{x}_{N+1}\right)$ that minimizes $\left\|\tilde{v}+H^{\prime}\right\|$ as follows. Compute the orthogonal projection $\bar{v}$ of $\tilde{v}$ onto $\mathscr{T}$, denoted by $\tilde{v}^{\prime}$, and let $L, P, Q$ and $\bar{x}(N+1)$ now denote the corresponding values of the parameters in
(22). Define $\Delta_{M}:=\left(\Delta_{A}, \Delta_{\tilde{B}}, \Delta_{C}, \Delta_{\bar{D}}\right)$ according to (20) by

$$
\left[\begin{array}{cc}
\Delta_{A} & \Delta_{\tilde{B}} \\
\Delta_{C} & \Delta_{\tilde{D}}
\end{array}\right]:=\left[\begin{array}{ccc}
A & B & \tilde{B} \\
C & D & \tilde{D}
\end{array}\right]\left[\begin{array}{cc}
0 & P \\
-L^{\mathrm{T}} & Q \\
-P^{\mathrm{T}} & 0
\end{array}\right] .
$$

Then $H^{\prime}\left(\Delta_{M}, \bar{x}_{N+1}\right)=\tilde{v}^{\prime}$, and $\left(-\Delta_{M},-\bar{x}_{N+1}\right)$ is the optimal variation. Redefine $(A, \tilde{B}, C, \tilde{D})$ as an ISR corresponding to $\mathscr{B}\left(A-\Delta_{A}, \widetilde{B}-\Delta_{\bar{B}}\right.$, $\left.C-\Delta_{C}, \tilde{D}-\Delta_{\tilde{D}}\right)$, and redefine $B$ and $D$ according to (17).

Step 4. If the variation $\Delta_{M}$ in (23) is zero then $\mathscr{B}(A, B, C, D)$ is a stationary point, so stop. If the misfit $d(w, \mathscr{B}(A, B, C, D))$ has decreased, repeat the algorithm for this model, otherwise first halve $L, P, Q$ and $\bar{x}(N+1)$ repeatedly, until the corresponding model improves the initial one.

Result. $\mathscr{B}(A, B, C, D)$ is a stationary point with respect to the GTLS criterion.

This algorithm has been implemented in Matlab. In addition to Algorithm 1, which is used in Steps 1 and 4, Step 3 involves the computation of an orthogonal projection in $\mathrm{R}^{p \times N}$ to determine the optimal variation, and solving a Riccati equation for the transformation to isometric form, as is described in Roorda and Heij (1995, Proposition 5.2).

\section{SIMULATION EXPERIMENT}

We illustrate the algorithms by a simple example derived from Roorda and Heij (1995). The data are generated as $w=w^{\prime}+e$, where $w^{\prime} \in \mathscr{B}^{3,1,2}$ satisfies the equations

$$
\begin{aligned}
& w_{2}^{\prime}(t)=w_{2}^{\prime}(t-1)+w_{1}^{\prime}(t), \\
& w_{3}^{\prime}(t)=w_{2}^{\prime}(t)+w_{1}^{\prime}(t-1) .
\end{aligned}
$$

For $w_{1}$ we take white noise with unit variance, and for $e$ a three-dimensional white noise process with independent components and variance $\sigma_{e}^{2}=0.01$. The observation interval has length 50. The simulation is implemented in the computer package Matlab.

First we apply Algorithm 1 to the system $\mathscr{B}_{\text {nom }}$ defined as the solution set of (24). This yields a decomposition $w=\hat{w}+\tilde{w}$, with $\hat{w} \in \mathscr{B}_{\text {nom }}$ and $\tilde{w}$ the corresponding approximation error. By definition of $\mathscr{B}_{\text {nom }}, \hat{w}$ satisfies the equations (24) for all $t \in\{2, \ldots, 50\}$; hence also all linear combinations of these equations are valid for $\hat{w}$. For example, substituting $w_{1}^{\prime}(t)=w_{2}^{\prime}(t)-$ $w_{2}^{\prime}(t-1)$, which follows from the first equation, 
into the second results in the second-order equation $\quad w_{3}^{\prime}(t)=w_{2}^{\prime}(t)+w_{2}^{\prime}(t-1)-w_{2}^{\prime}(t-2)$, which also holds for $\hat{w}$. The same holds for all higher-order implications of (24), which reflects the global character of the GTLS method. Consequently, $\tilde{w}$ has the interpretation of the minimal change in the data needed to make the system equations hold throughout the whole observation interval, which is in our opinion a convincing way to measure the accuracy of (24) for $w$.

One might expect that the error $\tilde{w}$ corresponding to the optimal approximation would be equal to the noise $e$ by which the observation has been corrupted, so that the squared misfit would be close to $E\|e\|^{2}=$ $3 \times 50 \times 0.1=1.5$. However, the misfit $d\left(w, \mathscr{B}_{\text {nom }}\right)$ turns out to be 0.96 , which is substantially smaller. This can be explained as follows. As the projection algorithm (Algorithm 1) is linear in the observation, the optimal approximation equals the sum of the optimal approximation of $w^{\prime}$ and of $e$. So $\hat{w}=w^{\prime}+\hat{e}$, with $\hat{e}$ the optimal approximation of $e$ in $\mathscr{B}_{\text {nom }}$, and $\tilde{w}=e-\hat{e}$. Without making precise statements about the size of $\hat{e}$, it is not hard to see, on the basis of (7), that $\hat{e} \neq 0$, which means that a part of the noise is incorporated in the approximation.

The recursive projection algorithm (Algorithm 2) gives insight into how the misfit is affected by each subsequent observation, given the values in the past. In Fig. 1 we compare the sequence $m(t)^{2}$, which is the increase of the squared misfit due to the observation $w(t)$, given its past, and $|\tilde{w}(t)|^{2}$, which is the squared misfit at time $t$ given the whole observation, as computed just before. This illustrates that the recursive procedure can be helpful for locating exceptional deviations in the data. For example, consider the results around $t=37$. The high peak in $m(t)^{2}$ at

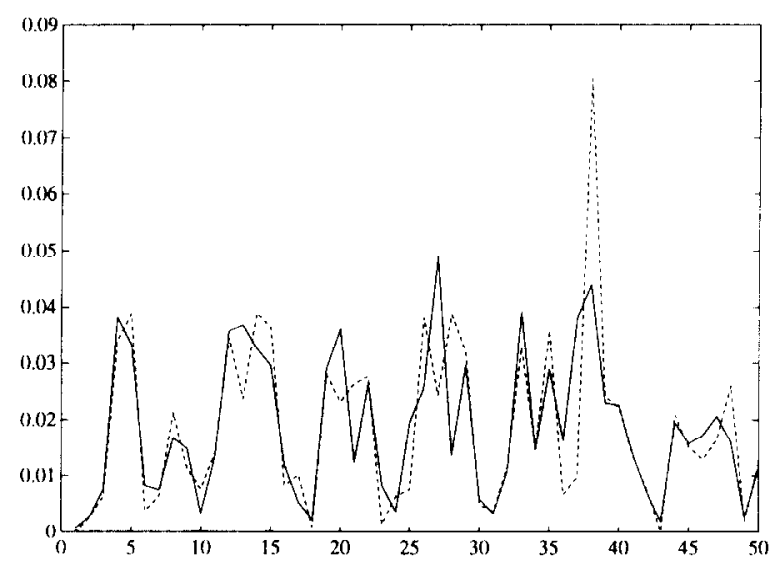

Fig. 1. Misfit sequences for observation $w$ in model $\mathscr{B}_{\text {nom: }}$; $-|\tilde{w}(t)|^{2} ; \cdots \cdots, m(t)^{2}$. $t=38$ clearly shows a relatively high deviation in the data at that point, while this is not obvious from the misfit sequence $|\tilde{w}(t)|^{2}$, since this shows a moderate error for both $w(37)$ and $w(38)$. So, although it appears to be optimal to distribute the deviation in $w(38)$ over several timeinstances afterwards, the recursive procedure clearly points out its exact location. Indeed, it turns out that the noise $e$ in the simulated data has a high peak at $t=38$.

Next we give an interpretation of the steady-state valuc of $\tilde{H}(t)$, denoted as $\tilde{H}:=$ $\lim \tilde{H}_{t}$. It turns out that $\tilde{H}$ has eigenvalues 0 , 0.56 and 0.97 with normalized orthogonal eigenvectors

$$
\left[\begin{array}{l}
0.58 \\
0.58 \\
0.58
\end{array}\right],\left[\begin{array}{r}
-0.68 \\
-0.06 \\
0.73
\end{array}\right],\left[\begin{array}{r}
0.45 \\
-0.81 \\
0.35
\end{array}\right],
$$

respectively. This gives a rather complete picture of the effect of an observation on the misfit, given the past. If $w(t)$ happens to be equal to $C \hat{x}_{t}(t)$ then $\epsilon(t):=w(t)-C \hat{x}_{t}(t)-0$, so the misfit does not increase. Moreover, $w(t)$ may vary in the direction of the kernel of $\tilde{H}$ without any cost, so the model leaves the level of $w(t)$ completely free. This is in accordance with (24), since the equations remain valid if the same constant is added to all three components of $w(t)$. In all other cases the misfit is increased. The ratio between the nonzero eigenvalues gives the relative severeness of these deviations with respect to the GTLS criterion.

Finally, we consider the construction of optimal GTLS models for $w$, with imposed complexity $(m, n)=(1,2)$. The misfit of the optimal model equals 0.93. As could be expected, this hardly improves the nominal model, and in fact the optimal model is quite close to the nominal one. To conclude, we compare the performance of the Gauss-Newton algorithm and the model improvement algorithm (Algorithm 3). Starting with $\mathscr{B}_{\text {nom }}$, the GaussNewton algorithm converges rapidly, with improvement below $10^{-15}$ within 14 steps, while for Algorithm 3 the improvement after 50 steps is above $4 \times 10^{-6}$ (Fig. 2).

The same pattern occurs if we start in a randomly chosen model, with each entry in the state representation $(A, B, C, D)$ the realization of independent white noise with unit variance. For 15 random initial models, the GaussNewton algorithm converged 11 times to the optimal model, of which 6 times were to an improvement below $10^{-15}$ within 25 steps, while Algorithm 3 converged 7 times to the optimum, 


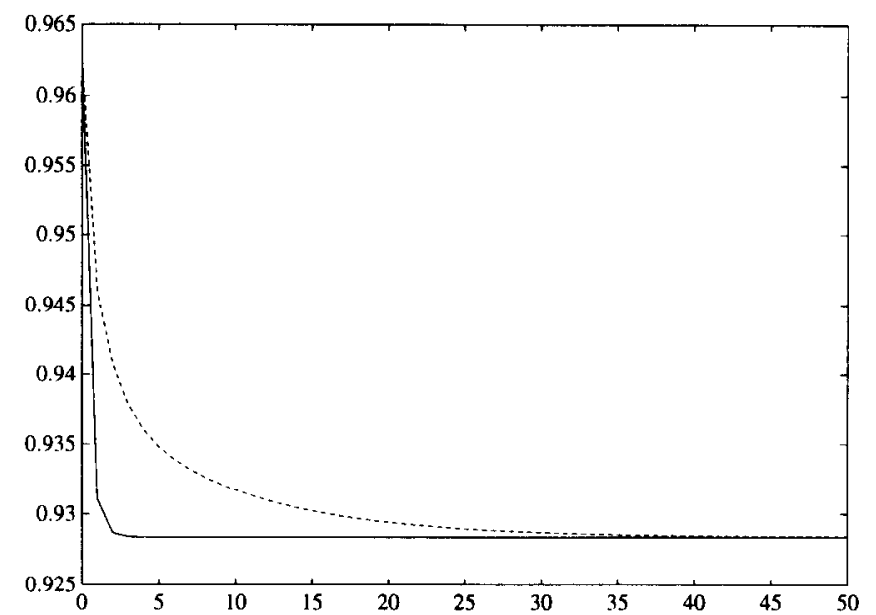

Fig. 2. Misfit in 50 iterations, starting in $\mathscr{B}_{\text {nom }}$, with the Gauss-Newton algorithm $(-$ ) and the model improvement algorithm (-.--).

with improvement above $10^{-5}$ after 50 steps. In some cases one or both algorithms converged to a locally optimal model, with misfit 1.45 .

\section{CONCLUSIONS}

We have presented three algorithms related to the global total least squares modelling of multivariate time series observed over a finite time interval. The first algorithm determines the optimal approximation of a time series, within the behaviour of a given system. The second does this recursively, which gives insight into the effect of the observations at each time, given their past. Thirdly, we have developed a Gauss-Newton algorithm for determining stationary points of the GTLS criterion, which appeared to be much faster than the algorithm described in Roorda and Heij (1995). We have also given a characterization of stationary points in terms of state representations. The algorithms have been illustrated by means of a simple example.

Acknowledgement-This research was supported in part by Grant SC1*-CT92-0779 on System Identification of the Science Program of the Commission of the European Community.

\section{REFERENCES}

Anderson, B. D. O. and J. B. Moore (1979). Optimal Filtering. Prentice-Hall, Englewood Cliffs, NJ.

Golub, G. H. and C. F. Van Loan (1983). Matrix Computations. Johns Hopkins University Press, Baltimore.

Roorda, B. and C. Heij (1995). Global total least squares modelling of multivariable time series. Discussion Paper 93-177, Tinbergen Institute, Erasmus University Rotterdam, The Netherlands. To appear in IEEE Trans. Autom. Control.

Willems, J. C. (1986a). From time series to linear system, part I: finite dimensional linear time invariant systems. Automatica, 22, 561-580.

Willems, J. C. (1986b). From time series to linear system, part II: exact modelling. Automutica, 22, 675-694.
Willems, J. C. (1987). From time series to linear system, part III: appropriate modelling. Automatica, 23, 87-115.

\section{APPENDIX-PROOFS}

Proof of Proposition 4.2. (Existence of ISR.)

The construction of ISRs described in Roorda and Heij (1995), Proposition 5.2, is also valid for stabilizable LTF systems.

Proof of Proposition 4.3. (Properties of ISR.)

For Parts (1) and (2) we refer to Roorda and Heij (1995). To give some intuition for Part (1), note that it follows from (6) that $A$ is stable. Further, it can be shown that state components corresponding to eigenvalues of $A$ on the unit circle are unobservable, and hence can be removed. Part (3) is obtained from $|\hat{x}(t+1)|^{2}+|\hat{w}(t)|^{2}=|\hat{x}(t)|^{2}+|\hat{v}(t)|^{2}$, which follows immediately from (6).

Proof of Proposition 5.1. (Orthogonal complement.)

The proof is based on the following result. Let $(\hat{x}, \hat{v})$ and $(\tilde{x}, \tilde{v})$ denote the state and auxiliary input for $\hat{w} \in$ $\mathscr{B}_{T}(A, B, C, D)$ and $\tilde{w} \in \mathscr{B}_{T}(A, \tilde{B}, C, \tilde{D})$, respectively, with $T=\{1, \ldots, N\}$. Then

$$
\langle\hat{w}, \tilde{w}\rangle=\hat{x}(1)^{\mathrm{T}} \tilde{x}(1)-\hat{x}(N+1)^{\mathrm{T}} \tilde{x}(N+1) .
$$

This can be proved as follows. Define

$$
\hat{x}:=\left[\begin{array}{c}
\hat{x} \\
\hat{v} \\
0
\end{array}\right] \text { and } \tilde{z}:=\left[\begin{array}{l}
\tilde{x} \\
0 \\
\tilde{v}
\end{array}\right] \text {, }
$$

so that $\tilde{w}=[C D \tilde{D}] \hat{z}$ and $\tilde{w}=[C D \tilde{D}] \tilde{z}$. Then it follows that

$$
\begin{aligned}
\langle\tilde{w}, \hat{w}\rangle & =\langle[C D \tilde{D}] \hat{z},[C D \tilde{D}] \tilde{z}\rangle=\left\langle[C D \tilde{D}]^{\mathrm{T}}[C D \tilde{D}] \hat{z}, \tilde{z}\right\rangle \\
& =\left\langle I-[A B \tilde{B}]^{\mathrm{T}}[A B \tilde{B}] \hat{z}, \tilde{z}\right\rangle=\langle\hat{z}, \tilde{z}\rangle-\langle[A B \tilde{B}] \hat{z},[A B \tilde{B}] \tilde{z}\rangle \\
& =\sum_{t=1}^{N} \hat{x}(t)^{\mathrm{T}} \tilde{x}(t)-\hat{x}(t+1)^{\mathrm{T}} \tilde{x}(t+1),
\end{aligned}
$$

from which (A.1) follows.

Now let $\mathscr{V}$ denote the space $\{\tilde{w}: T \rightarrow$ $\left.\mathrm{R}^{q} ;\{\ldots, 0,0, \tilde{w}, 0,0, \ldots\} \in \mathscr{B}(A, \tilde{B}, C, \tilde{D})\right\} ;$ so elements of $\mathscr{V}$ correspond to system trajectories in $\mathscr{B}(A, \tilde{B}, C, \tilde{D})$ with 
boundary states $x(1)=0$ and $\hat{x}(N+1)=0$. From $(A .1)$, it follows that $\mathscr{V} \subset\left(\mathscr{B}_{T}\right)^{\perp}$. Finally, $\mathscr{V}=\left(\mathscr{B}_{T}\right)^{\perp}$ follows from

$$
\begin{aligned}
\operatorname{dim}\left(\mathscr{B}_{T}\right)^{2} & =\operatorname{dim}\left(\mathrm{R}^{q}\right)^{\mathrm{T}}-\operatorname{dim}\left(\mathscr{B}_{T}\right) \\
& =q(N+1)-m(N+1)-n=(q-m)(N+1)-n \\
& =\operatorname{dim}(\eta) .
\end{aligned}
$$

Proof of Algorithm 1. (Optimal approximation in a given system.)

First we remark that Step 2 is easily implemented, since $\tilde{v}$ depends linearly on the end state $x(N+1)$. Moreover, we give an explicit, recursive formula for the end state in Algorithm 2.

In view of Proposition 5.1, it remains to prove that

(1) $\hat{w}+\vec{w}-w$.

(2) $\hat{w} \in \mathscr{B}$,

(3) $\tilde{w} \in\left(B_{T}\right)^{\mathrm{i}}$

(4) $\|\tilde{w}\|=\|\tilde{v}\|$.

(1) By premultiplying the equations (7) by $\left[\begin{array}{ccc}A & B & \tilde{B} \\ C & D & \tilde{D}\end{array}\right]$ it is easily shown that $\hat{x}+\bar{x}=x$ and $\hat{w}+\tilde{w}=n$

(2) $w \in \mathscr{B}_{T}$ by construction.

(3) $\bar{w} \in \mathscr{B}_{T}(A, \tilde{B}, C, \tilde{D})$ and $\tilde{x}(1)-0$ by construction. In view of Proposition 5.1, it remains to prove that $\tilde{x}(N+1)=0$. Since in Step 2, $x(N+1)$ is chosen such that $\tilde{v}$ is minimal, $\tilde{v}$ must be orthogonal to the effect of varying the end state $x(N+1)$ in Step 2. Hence for all $x \in \mathrm{R}^{n}, \quad \sum_{k}^{N} x^{\mathrm{T}} A^{N \cdot k} \tilde{B} \tilde{v}(k)=0, \quad$ so $\quad \sum_{k=1}^{N} A^{N \cdot k} \tilde{B} \tilde{v}(k)=$ $\tilde{x}(N+1)=0$.

(4) This follows from Proposition 4.3(3) and the fact that $(A, \tilde{B}, C, \tilde{D})$ is isometric.

Proof of Algorithm 2. (Recursive approximation in a given system.)

The main part of this proof is devoted to the state update equation (12). Once this has been established, the formula for the past-induced approximation $\hat{w}_{i}(t)$ in (13) and the formulas for the misfit follow from straightforward calculations.

We shall derive (12) by induction. In principle, we could start at $t=0$, but note that $\bar{x}_{0}(1)$ is a meaningless variable since it denotes the optimal end state of an empty observation. In fact, it can be shown that the value for $\hat{x}_{10}(1)$ is irrelevant, since it has no effect on the evolution of $\hat{x}_{i}(t+1)$ in (12). However, it might be more clarifying to start from the inductive proof at $t=1$.

From Algorithm 1, it follows that $x_{1}(2)$ should minimize $\|\tilde{v}(1)\|^{2}=\left\|\widetilde{B}^{\mathrm{T}} x_{1}(2)+\tilde{D}^{\mathrm{T}} w(1)\right\|^{2}$. Taking the derivative after $x_{1}(2)$ gives $\tilde{B} \tilde{B}^{\mathrm{T}} x_{1}(2)+\tilde{B} \tilde{D}^{\mathrm{T}} w(1)=0=\tilde{W}_{2} x_{1}(2)+$ $\tilde{F}_{1} w(1)$. If $\tilde{W}_{2}$ is invertible, it follows that $x_{1}(2)=\tilde{W}_{2}{ }^{\prime} \tilde{F}_{1} w(t)$ is indeed optimal. For the case that $\tilde{W}_{2}$ is singular, we need the following lemma.

Lemma A.1. (Pseudo-inverse.) Consider the equation $W x+z=0$, with $W$ singular, and $z \in \operatorname{im} W$. Let $W^{\#}$ denote a pseudo-inverse of $W$, i.e. $W^{\#} W W^{\#}=W^{\#}$ and $W W^{\sharp} W=W$. Ihen $x=-W^{H} z$ is a solution.

Proof. As $z \in \operatorname{im} W$, there exists a $z^{\prime}$ such that $z=W z^{\prime}$ Substituting $x=-W^{\#} z$ gives $W x+z=-W W^{\#} W z^{\prime}+W z^{\prime}=$ 0 .

This lemma can be applied to the equation $W_{1} x_{1}(2)+$ $\tilde{F}_{1} w(1)=0$, since $\tilde{F}_{1} w(1)=\tilde{B} \tilde{D}^{\mathrm{T}} w(1) \subset \mathrm{im} \tilde{W}_{2}$, which gives $x_{1}(2)=\tilde{W}_{2}^{\#} \tilde{F}_{1} w(t)$.

Thus we have proved (12) for $t=1$. The induction step is proved with the aid of the following lemma.

Lemma A.2. Suppose $x_{i}{ }_{1}(t)$ minimizes $\left\|\tilde{v}_{,},{ }_{1}\right\|$ in (7) with $N=t-1$. Then $x_{t}(t+1)$ minimizes $\left\|\tilde{v}_{t}\right\|$ in (7) with $N=t$ if and only if

$$
\bar{W}_{t, 1} x_{t}(t+1)=\bar{W}_{t+1} A x_{,}{ }_{1}(t)-\tilde{F}_{t} \epsilon(t) .
$$

Proof. Define $\bar{x}(t):=x_{t}(t)-x_{i, 1}(t)$, denoting the adaption of $x$ at time $t$ due to the observation $w(t)$. Similarly, define $\bar{v}\left(t^{\prime}\right):=\tilde{v}_{i}\left(t^{\prime}\right)-\tilde{v}_{t-1}\left(t^{\prime}\right)$. From the definition of $\tilde{v}_{t, 1}$ and $\bar{v}_{i}(\mathrm{cf} .(7))$, it follows that $\bar{v}(t)$ equals the backward effect of $\vec{x}(t)$ in $(7)$, i.e.

$$
\left.v(t):=\tilde{v}_{t}\left(t^{\prime}\right)-\tilde{v}_{t}, t^{\prime}\right)=\tilde{B}^{\top} A^{\mathrm{I}^{\prime} t^{\prime}}{ }^{\prime} \bar{x}(t) \text { for } 1 \leq t^{\prime} \leq t-1 .
$$

From the definition of $\tilde{W}_{t}$, it follows that $\|\tilde{v}\|=\left[\bar{x}^{\mathrm{T}} \tilde{W}_{t} \bar{x}(t)\right]^{1 / 2}$ Moreover, $v$ is orthogonal to $\bar{v}, 1$ (cf. the proof of Algorithm 1(3)). This gives

$$
\left\|\tilde{v}_{t}\right\|^{2}=\left\|\tilde{v}_{t, 1}\right\|^{2}+\bar{x}^{\mathrm{T}} \tilde{W}_{r} \bar{x}(t)+\left|\tilde{v}_{t}(t)\right|^{2} .
$$

So $x_{t}(t+1)$ minimizes $\left\|\tilde{v}_{t}\right\|$ if and only if it minimizes $\bar{x}^{1} \tilde{W}, \bar{x}(t)+\left|\tilde{v}_{t}(t)\right|^{2}$. Substituting $\tilde{v}_{t}=\tilde{B}^{1} x_{t}(t+1)+\bar{D}^{1} w(t)$ and $\bar{x}(t)=A^{\mathrm{T}} x_{t}(t+1)+C^{\mathrm{T}} w(t)-x_{t}{ }_{1}(t)$, and then taking the derivative after $x_{1}(t+1)$ gives the result.

If $\vec{W}_{t, 1}$ is invertible, (12) follows immediately. If $\tilde{W}_{t+1}$ is singular, we have to replace the inverse $\tilde{W}_{t+1}$ by a pseudo-inverse $\tilde{W}_{t+1}^{\#}$. Note that $\operatorname{im} \tilde{F}_{t} \subset \operatorname{im} \tilde{W}_{t+1}$, since

$$
\begin{aligned}
& \tilde{W}_{t+1}=\left[\begin{array}{ll}
A \tilde{W}_{t}^{1 / 2} & \tilde{B}
\end{array}\right]\left[\begin{array}{ll}
A \tilde{W}_{!}^{1 / 2} & \tilde{B}
\end{array}\right]^{\mathrm{T}} . \\
& \tilde{F}_{t}=\left[\begin{array}{ll}
A \tilde{W}_{t}^{1 / 2} & \tilde{B}
\end{array}\right]\left[C \tilde{W}_{t}^{1 / 2} \quad D\right]^{\top} .
\end{aligned}
$$

Now the result follows from applying Lemma A.1. This concludes the inductive proof of (12).

Next we prove (13), by showing that

$$
\bar{w},(t)=\tilde{H}, \epsilon(t)
$$

Then the result follows from $\hat{w}_{t}(t)=w(t)-\tilde{w}_{t}(t)=C \hat{x}_{t}(t)+$ $\left(I-\tilde{H}_{t}\right) \epsilon(t)$, where we have used the definition of $\epsilon,(11)$ By definition, $\tilde{w}_{t}(t)=C \tilde{x}_{t}(t)+\tilde{D} \tilde{v}_{t}(t)$, where

$$
\begin{aligned}
\tilde{x}_{t}(t) & =\sum_{k=1}^{t} A^{k} \tilde{B} \tilde{v}_{t}(t-k) \\
& =\sum_{k=1}^{t} A^{k} \tilde{B} \bar{v}_{t}(t-k)+\sum_{k=1}^{t} A^{k} \tilde{B} \tilde{v}_{t-1}(t-k) \\
& =\tilde{W}_{t} \bar{x}_{t-1}+\tilde{x}_{t-1}(t)=\tilde{W}_{t} \bar{x}_{t-1},
\end{aligned}
$$

where we have used (27) to obtain the third equation, while $\tilde{x}_{t, 1}(t)=0$ follows from Proposition 5.1. So $\tilde{w}(t)=$ $C \tilde{W}_{t} \bar{x}_{t}(t)+\tilde{D} \tilde{v}_{t}(t)$. Now substituting $\bar{x}(t)=x_{t}(t)-x_{t-1}(t)$ and

$$
\begin{aligned}
& x_{t}(t)=A^{\mathrm{T}} x_{t}(t+1)+C^{\mathrm{T}} w(t), \\
& v_{t}(t)=\tilde{B}^{\mathrm{T}} x_{t}(t+1)+\tilde{D}^{\mathrm{T}} w(t)
\end{aligned}
$$

gives $\tilde{w}_{t}(t)=\tilde{F}_{t}^{\mathrm{T}} x_{t}(t+1)+\tilde{G}_{t} w(t)-C \tilde{W} x_{t}(t)$. Using (12) gives

$$
\tilde{w}_{t}(t)=\tilde{H}_{t} \epsilon(t)+\left(\tilde{F}_{t} A+\tilde{G}_{t} C-C \tilde{W}_{t}\right) x_{t-1}(t) .
$$

It is easily derived from (6) that the last term cancels, from which (A.5) follows.

Finally, we have to prove the equations for the misfit From (A.4), it follows that

$$
m(t)^{2}=\left|\bar{v}_{t}(t)\right|^{2}+\bar{x}(t)^{\mathrm{r}} \tilde{W} \bar{x}_{i}
$$

Rewrite $\bar{x}(t)$ as

$$
\begin{aligned}
& \bar{x}(t)=A^{\mathrm{T}} x_{t}(t+1)+C^{\mathrm{T}} w(t)-x_{t}{ }_{1}(t) \text { from (30) } \\
& \therefore A^{\mathrm{T}} x_{t}(t+1)+C^{\mathrm{T}} \epsilon(t)+C^{\mathrm{l}} C x_{t-1}(t) \text { from (11) } \\
& =\left(A^{\prime} A+C^{1} C-I\right) x_{t}(t+1)+\left(C^{1}-A^{\top} \bar{W}_{t+1}^{1} \tilde{F}_{t}\right) \epsilon(t) \\
& =\left(C^{\mathrm{I}}-A^{\mathrm{T}} \tilde{W}_{t+1}{ }_{1} \tilde{F}_{t}\right) \epsilon(t) \text { from }(6) .
\end{aligned}
$$$$
\text { from (12) (A.11) }
$$

Further, rewrite $\tilde{v}_{t}(t)=\left(\tilde{D}^{\mathrm{T}}-\tilde{B}^{\mathrm{T}} \tilde{W}_{t+1}{ }^{1} \tilde{F}_{t}\right) \epsilon_{t}$, which is ob- 
tained similarly. Substituting these formulas for $\bar{x}(t)$ and $\tilde{v}_{t}(t)$ into (A.8) gives the result for $m(t)$.

The formula for $m_{0}(t)$ is directly obtained from (A.5). Finally, the equation for $m_{-}(t)$ follows from $m(t)^{2}=$ $m_{-}(t)^{2}+m_{1}(t)^{2}$.

Proof of Algorithm 3. (Model improvement algorithm.)

For a proof we refer to Roorda and Heij (1995, Theorems $7.2,8.1$ and 8.2). The only difference with the algorithm presented there is that we need to optimize the initial state $\hat{x}(1)$ of the approximation, since we now consider observations over finite time, but the proof is easily adapted for this. Concerning the implementation of the algorithm, we make the following remarks. In each step optimal values of some parameters are determined, keeping the remaining ones fixed, which leads to a sequence of decreasing misfits. Notice that Steps 2 and 3 are quadratic in the parameters, and hence are easily solved. Step 4 involves the singular value decomposition of $\hat{v}$ (cf. Golub and Van Loan, 1983).

Proof of Theorem 7.2. (Optimality conditions.)

The proof is completely analogous to the proof for time series in $l_{2}$ (cf. Roorda and Heij, 1995).

Proof of Proposition 8.1. (GTLS as minimization over ISRs.)

The proof is entirely based on Algorithm 1. Define $B$ and $D$ according to the first step. The auxiliary input $\tilde{v}$ of the approximation error, as defined in (7), equals $H\left((A, \tilde{B}, C, \tilde{D}), x_{N+1}\right)$ if $x_{N+1}$ minimizes $\|\tilde{v}\|$ (cf. Step 2). As $d(w, \mathscr{B}(A, B, C, D))=\|\tilde{v}\|$, it follows that $\mathscr{B}(A, B, C, D)$ is an optimal model for $w$ if and only if the minimum of $\|H\|$ is achieved for $\left((A, \tilde{B}, C, \tilde{D}), x_{N+1}\right)$.

Proof of Lemma 8.2.

The space $U$ of unitary $k \times k$ matrices is a differentiable manifold in $\mathbf{R}^{k \times k}$ of dimension $\frac{1}{2} k(k-1)$, so the tangent space $T_{M} U$ of $U$ at a point $m$ is a linear space of this dimension, contained in $\left\{M K ; K \in \mathrm{R}^{k \times k}\right\}$. Now it is easily verified that $M K \in T_{M} \mathscr{U}$ if and only if $K+K^{\mathrm{T}}=0$, by considering the fact that

$$
(M+M K)^{\mathrm{T}}(M+M K)=I+K^{\mathrm{T}}+K+K^{\mathrm{T}} K .
$$

Proof of Algorithm 4. (Gauss-Newton for GTLS.) We shall prove that:

(1) the formula (21) for the derivative of $H$ is correct;

(2) $\left\|\tilde{v}+H^{\prime}\right\|$ is minimized for the variation given in (23)

(3) if $\Delta_{M}=0$ in Step 4 then the model is a stationary point;

(4) different variations of the form (20) are not equivalent if $(A, B, C, D)$ is minimal.

Then the convergence to stationary points follows from the general considerations underlying Gauss-Newton algorithms. The main issue is to prove that the second point holds despite the fact that we have restricted the variations of ISRs to the form (20). Further, the fourth point implies that generically we cannot further reduce the number of variations.

(1) From straightforward calculations, it follows that for $\Delta_{M}:=\left(\Delta_{A}, \Delta_{\bar{B}}, \Delta_{C}, \Delta_{\tilde{D}}\right)$ the derivative $H^{\prime}\left(\Delta_{M}, \bar{x}_{N+1}\right)=: \bar{v}$ of $H$ is given by

$$
\begin{aligned}
& \bar{x}(t)=A^{\mathrm{T}} \bar{x}(t+1)+\Delta_{A}^{\mathrm{T}} x(t+1)+\Delta_{C}^{\mathrm{T}} w(t), \\
& \text { with } \bar{x}(N+1)=\bar{x}_{N+1}, \\
& \bar{v}(t)=\tilde{B}^{\mathrm{T}} \bar{x}(t+1)+\Delta_{\bar{B}}^{\mathrm{T}} x(t+1)+\Delta_{\bar{D}}^{\mathrm{T}} w(t) .
\end{aligned}
$$

Substituting (20) and using (7) gives the result.

(2) Define $\tilde{V}:=\operatorname{im} H$. Then im $H^{\prime}=T_{\bar{v}} \tilde{V}$. Clearly, $\| \bar{v}+$ $H^{\prime}\left(\Delta_{M}, \bar{x}_{N+1}\right) \|$ is minimized for $H^{\prime}\left(\Delta_{M}, \bar{x}_{N+1}\right)=-\tilde{v}^{*}$, with

† By definition, the derivative of $H$ is given by $H^{\prime}: T_{(M, x)}{ }^{\natural} \rightarrow \mathrm{R}^{p \times N}$, which assigns to every direction $\left(\Delta_{M}, \bar{x}(N+1)\right)$ the directional derivative of $H$, i.e. $H_{(M, x)}^{\prime}\left(\Delta_{M}, \bar{x}(N+1)\right):=\partial H(c(t)) /\left.\partial t\right|_{i=0}$, with $c:(-\epsilon, \epsilon) \rightarrow$ $u_{n+q} \times \mathbf{R}^{n}$ a differentiable curve with $c(0)=(M, x)$ and $c^{\prime}(0)=\left(\Delta_{M}, \bar{x}(N+1)\right)$ $\tilde{v}^{*}$ the orthogonal projection of $\tilde{v}$ onto $T_{\tilde{v}} \tilde{V}$. We shall prove that

$$
T_{\tilde{v}} \tilde{V}=\mathscr{T}+\mathscr{R}, \quad \text { with } \mathscr{R} \perp \tilde{\boldsymbol{v}} .
$$

Then the orthogonal projections $\bar{v}$ onto $T_{\tilde{v}} \bar{V}$ and onto $\mathscr{T}$ coincide, i.e. $\tilde{v}^{\prime}=\tilde{v}^{*}$, so that substracting the variation (23) corresponding to $\tilde{v}^{\prime}$ is indeed optimal. So it remains to prove (A.14). Therefore, we compare the definitions of $T_{\tilde{v}} \tilde{V}$ and $\mathscr{T}$. By definition, $\quad T_{\tilde{u}} \tilde{V}=\operatorname{im} H^{\prime}=\left\{H^{\prime}\left(\Delta_{M}, \bar{x}_{N+1}\right) ; \quad \Delta_{M} \in\right.$ $\left.T_{M} \mathscr{I}, \bar{x}_{N+1} \in \mathrm{R}^{n}\right\}$. According to Lemma 8.2, $T_{M} \mathscr{I}$ consists of variations $\left(\Delta_{A}, \Delta_{\tilde{B}}, \Delta_{C}, \Delta_{\tilde{D}}\right)$ of the following form (in order to preserve the symmetry in the formulas, we also describe the corresponding variations of $B$ and $D$ :

$$
\left[\begin{array}{ccc}
\Delta_{A} & \Delta_{B} & \Delta_{\tilde{B}} \\
\Delta_{C} & \Delta_{D} & \Delta_{\tilde{D}}
\end{array}\right]=\left[\begin{array}{lll}
A & B & \tilde{B} \\
C & D & \tilde{D}
\end{array}\right]\left[\begin{array}{ccc}
X & L & P \\
-L^{\mathrm{T}} & Y & Q \\
-P^{\mathrm{T}} & -Q^{\mathrm{T}} & R
\end{array}\right],
$$

with $X, Y$ and $R$ antisymmetric. Note that $\mathscr{T}$ is defined similarly to $T_{\tilde{v}} \tilde{V}$, but with the variations restricted to $(20)$. So it remains to show that by taking the diagonal blocks in (A.15) equal to zero we only remove some directions $\mathscr{R}$ in $T_{\tilde{v}} \tilde{V}$ that are orthogonal to $\tilde{v}$. Such directions are irrelevant in the Gauss-Newton algorithm, as explained before. In fact, we shall show that, roughly speaking, $R$ corresponds to a unitary basis transformation of the auxiliary input $\tilde{\boldsymbol{v}}$, leaving $\| \tilde{v} \mid$ unchanged, while the effect of $x$ in (A.15) can always be compensated for by an appropriate unitary basis transformation of the state space.

First we analyse the effect of $R$ in (A.15), i.e. we consider variations of the form $\Delta_{M}=\left(\Delta_{A}, \Delta_{\tilde{B}}, \Delta_{C}, \Delta_{\tilde{D}}\right)=$ $(0, \tilde{B} R, 0, \tilde{D} R)$ with $R$ antisymmetric. This is nothing other than a variation corresponding to a unitary basis transformation of the auxiliary input $\tilde{v}$ (cf. Proposition 4.3(2) and Lemma 8.2). For this variation, (A.13) gives $H^{\prime}\left(\Delta_{M}, 0\right)=\bar{v}$, with $\bar{v}(t)=R^{\mathrm{T}}[\tilde{B} x(t+1)+\tilde{D} w(t)]=R^{\mathrm{T}} \tilde{v}(t)$. Now define

$$
\mathscr{R}:=\left\{R^{\mathrm{T}} \tilde{v} ; R+R^{\mathrm{T}}=0\right\} .
$$

Then $\mathscr{R} \perp \tilde{v}$, since $\left\langle R^{\mathrm{T}} \tilde{v}, \tilde{v}\right\rangle=\langle\tilde{v}, R \tilde{v}\rangle=-\left\langle\tilde{v}, R^{\mathrm{T}} \tilde{v}\right\rangle=0$. This reflects the fact that a unitary basis transformation of $\tilde{v}$ has no effect on $\|\tilde{\boldsymbol{v}}\|$.

Finally, we shall prove that, without loss of generality, we can take $X=0$ in (A.15), by showing that all elements in $T_{\tilde{v}} \tilde{V}$ can be generated with $X=0$. Note that a basis transformation of the state space does not change the value of $H$; hence $H^{\prime}$ is a singular mapping since $H^{\prime}\left(\Delta_{M}, \vec{x}_{N+1}\right)=0$ for $\Delta_{M}$, a variation corresponding to a unitary state space transformation, and $\bar{x}_{N+1}$, the corresponding change in the end state. The structure of these variations is determined as follows. To preserve symmetry in the formulas, we again consider variations of $B$ and $D$. Define

$$
\bar{B}:=\left[\begin{array}{ll}
B & \tilde{B}
\end{array}\right], \quad \bar{D}:=\left[\begin{array}{ll}
D & \tilde{D}
\end{array}\right],
$$

and define $\Delta_{\bar{B}}$ and $\Delta_{\bar{D}}$ accordingly. The linearization of the mapping

$$
U \rightarrow\left(U A U^{\mathrm{T}}, U B, C U^{\mathrm{r}}, D\right)
$$

around $U=I_{p}$ is given by

$$
I+\Delta_{U} \rightarrow\left(\Delta_{U} A+A \Delta_{U}^{\mathrm{T}}, \Delta_{U} B, C \Delta_{U}^{\mathrm{T}}, 0\right),
$$

so it follows from Lemma 8.2 that unitary state space transformations correspond to variations $\left(\Delta_{A}, \Delta_{\bar{B}}, \Delta_{C}, \Delta_{\bar{D}}\right)=$ $\left(\Delta_{I} A-A \Delta_{I J}, \Delta_{I} \bar{B},-C \Delta_{U}, 0\right)$ with $\Delta_{U}+\Delta_{U}^{\mathrm{T}}=0$. Rewriting this according to (A.15) gives

$$
\left[\begin{array}{cc}
\Delta_{A} & \Delta_{\bar{B}} \\
\Delta_{C} & \Delta_{\bar{D}}
\end{array}\right]=\left[\begin{array}{cc}
A & \bar{B} \\
C & \bar{D}
\end{array}\right]\left[\begin{array}{cc}
A^{\mathrm{T}} \Delta_{U} A-\Delta_{U} & A^{\mathrm{T}} \Delta_{U} \bar{B} \\
A^{\mathrm{T}} \Delta_{U} \bar{B} & \bar{B}^{\mathrm{T}} \Delta_{U} \bar{B}
\end{array}\right] .
$$

As transformations of the state space do not affect $H$, it follows that these variations belong to the kernel of $H^{\prime}$, taking $\bar{x}_{N+1}=\Delta_{U} x(N+1)$. This can also be proved directly, by substituting (A.17) into (A.13), which gives $\bar{x}(t)=\Delta_{U} x(t)$ and $\vec{v}(t)=0$ for $t \in\{1, \ldots, N\}$

Now define for a variation of the form (A.15), $\Delta_{u}$ such that

$$
X-A^{\mathrm{T}} \Delta_{u} A-\Delta_{u}=0, \quad \Delta_{u}+\Delta_{u}^{\mathrm{T}}=0,
$$


and subtract the corresponding variation (A.17). This results in an equivalent variation with zero value for the $X$-block in (A.15). Notice that for minimal ISRs, $A$ is asymptotically stable (cf. Proposition 4.3(1)). Then the solution is given by $\Delta_{U}:=-\sum_{k=1}^{\infty} A^{k} K_{1} A^{\mathrm{T}^{k}}$.

So there is only one detail left, concerning the case where $A$ is not asymptotically stable. Note that there might be no solution for (A.18) in this case. However, then $(A, B, C, D)$ is not minimal, and there is an additional source of non-uniqueness beside the choice of the unitary transformations as described in Proposition 4.3(2), which can be seen as follows. As $A$ is stable (cf. Proposition 4.3(1)), it must have some poles on the unit circle if it is not also asymptotically stable. Together with the isometry property, this implies that the matrix in (17) can be transformed by a unitary state space transformation to the form

$$
\left[\begin{array}{ccc}
A_{0} & 0 & 0 \\
0 & A_{\ldots} & \tilde{B}^{\prime} \\
0 & C^{\prime} & \tilde{D}^{\prime}
\end{array}\right],
$$

with $A_{0}$ unitary and $A_{-}$asymptotically stable. As the state components corresponding to $A_{0}$ are completely ineffective, we can replace $A_{0}$ by an arbitrary unitary matrix, or, equivalently, we can apply the transformation $A_{0} L$ with $L$ unitary. Consequently, in addition to (A.17), the variations corresponding to this transformation are also contained in the kernel of $H^{\prime}$. Using this extra freedom, we can obtain an equivalent variation with $X=0$, even if $A$ is not asymptotically stable. As this concerns only a non-generic case, we leave the details to the reader.

(3) If $\Delta_{m}=0$, it follows that also $\bar{x}_{N+1}=0$, since $x_{N+1}$ is optimal for $(A, \tilde{B}, C, \tilde{D})$ (cf. Step 1). This implies that $\left\|\tilde{v}+H^{\prime}\right\|$ is minimized for $\left(\Delta_{M}, \bar{x}_{N+1}\right)=(0,0)$ (cf. Part (1)), which means that the derivative of $\|H\|$ is zero in $\tilde{v}$; hence $\left((A, \tilde{B}, C, \tilde{D}), x_{N+1}\right)$ is a stationary point of $H$.

(4) It is easily verified that the reduction of the number of parameters in the variations (A.15) by taking the antisymmetric diagonal blocks $X=0$ and $R=0$ equals $\frac{1}{2} n(n-1)+\frac{1}{2} p(p-1)$, with $p:=q-m$. This is exactly the number of free parameters in the equivalent transformation of $(A, \tilde{B}, C, \tilde{D})$ as described in Proposition 4.3(2). As for minimal ISRs, this is the only source of non-uniqueness and it follows that different variations in (20) cannot be equivalent. 